\title{
Towards the interaction between large solutes in a fluid of small hard spheres
}

\author{
D.Henderson ${ }^{1}$, D.T.Wasan ${ }^{2}$, A.Trokhymchuk ${ }^{1,3}$ \\ 1 Department of Chemistry and Biochemistry, Brigham Young University, \\ Provo, UT 84602, USA \\ 2 Department of Chemical and Environmental Engineering, \\ Illinois Institute of Technology, \\ Chicago, IL 60616, USA \\ 3 Institute for Condensed Matter Physics \\ of the National Academy of Sciences of Ukraine, \\ 1 Svientsitskii Str., 79011 Lviv, Ukraine
}

Received September 25, 2001

The equivalence of the asymptotic expression for the interaction energy between a pair of large hard spheres in a fluid of small hard spheres that has been obtained by Roth et al. [Phys. Rev. E, 2000, vol. 62, p. 5360] using Fourier transforms and an expression that we have obtained [J. Colloid Int. Sci., 1999, vol. 210, p. 320] using Laplace transforms is pointed out and commented upon.

Key words: colloidal suspensions, interaction

PACS: 82.70.Dd, 61.20.Gy

\section{Introduction}

This paper is dedicated to Jean-Pierre Badiali on the occasion of his 60th birthday. Jean-Pierre is a generous friend who has made many important contributions to the theory of electrolytes and their interfaces. The authors wish him many more productive, healthy, and happy years.

In their paper on the depletion potential in hard-sphere mixtures, Roth et al. [1] have reported results for the asymptotic form of the interaction potential between two large spheres in a fluid of small spheres. Recently [2-4], we have developed simple analytic expressions for the force and interaction potential for the same system. Our result is based on the asymptotic form of these functions, as obtained from the zeros of the Laplace transform solution of the Ornstein-Zernike equation [2,5]. The result of Roth et al. is obtained from the zeros of the Fourier transform of the OrnsteinZernike relation between pair and direct correlation functions [6]. Quite obviously, 
the Laplace and Fourier transform techniques must lead to the same results. Indeed, the Fourier transform is related to the Laplace transform with an argument that is complex. The purpose of this note is to draw attention to the equivalence of the two results and make a few comments and some generalizations.

\section{Consideration}

Roth et al. [1] comment on the fact that for a binary hard-sphere mixture in the dilute limit of the large (radii $R$ ) spheres, the denominator of the Fourier transform of the small-small (ss), large-small (ls) and large-large (ll) total correlation functions is the same and equal to that of the fluid of pure small (diameter $\sigma$ ) spheres,

$$
\hat{D}(q)=1-\rho \hat{c}(q)
$$

with $\hat{c}(q)$ referring to the Fourier transform of the direct correlation function of the fluid of pure small particles, and $\rho$ is the number density of the small particles. Henderson [5] had already noted this using the Laplace transforms. Following the Percus-Yevick (PY) theory, in the limit of infinite dilution of large spheres, the denominator of the Laplace transforms of all three total correlation functions is

$$
P(s)=L(s)+\mathrm{e}^{s} S(s)
$$

where $L(s)$ and $S(s)$ are two polynomials, first defined by Wertheim [7]. This result is not as remarkable as Roth et al. feel. Since the large spheres are present in extreme dilution, the basic behaviour of all the correlations in the system, which is governed by the pole structure of the Fourier transform or Laplace transform, must be that for a fluid of small spheres. This is valid in general and presumes that the same approximation is used throughout.

The pole structure of the total correlation functions is determined by the zeroes of $P(s)$ or $\hat{D}(q)$. In general, there are an infinite number of solutions for $P(s)=0$ or $\hat{D}(q)=0$, but the asymptotic behaviour is governed only by the zero with the smallest imaginary part. Such a zero of $P(s)$ in the form $s=\kappa+\mathrm{i} \omega$ has been tabulated as a function of the packing fraction of the small spheres by Perry and Throop [8] in their calculations of the Percus-Yevick asymptotic form of the total correlation function for a one-component hard-sphere fluid. The zero of $\hat{D}(q)$ in the form $q=$ $a_{1}+\mathrm{i} a_{0}$ has been analyzed by Roth et al. [1]. Although both zeroes have been parametrized by slightly different analytical functions $[1,2]$, their numerical values are the same. The resulting expression is an exponentially damped trigonometric function

$$
h_{s s}^{\mathrm{PY}}(r) \sim\left(A_{s s} / r\right) \cos \left(\omega r+\varphi_{s s}\right) \exp (-\kappa r),
$$

where $A_{s s}$ and $\varphi_{s s}$ are the amplitude and the phase parameters, respectively. It has been already pointed $[2,6]$ that asymptotic (2.3) reproduces the Percus-Yevick solution not only at very large separations but is remarkably accurate down to the second nearest-neighbour shell. 
The decay of the Percus-Yevick total correlations, $h_{l l}^{\mathrm{PY}}(r)$, between two large spheres is of the similar form as given by equation (2.3) only with the new amplitude and phase parameters, $A_{l l}$ and $\varphi_{l l}$. The function $h_{l l}^{\mathrm{PY}}(r)$ might be used to calculate the interaction $W(r)$ between two large solutes through the exact relation

$$
\beta W(x)=-\ln \left[1+h_{l l}(x)\right],
$$

where $x=r-R-\sigma / 2$. However, it has been argued [9] that if $2 R \gg \sigma$, the Percus-Yevick theory for total correlations between large spheres being applied in equation (2.4), i.e. assuming $h_{l l}=h_{l l}^{\mathrm{PY}}$, leads to the result which does not obey the experimentally observed law: $W \sim R$ [10]. A qualitatively correct approximation for the total correlations between two large spheres which still involves Percus-Yevick theory has the form [5]

$$
1+h_{l l}(x)=\exp \left[h_{l l}^{\mathrm{PY}}(x)\right] .
$$

For the potential of mean force between two large spheres this leads to [2]

$$
\beta W(x)=-h_{l l}^{\mathrm{PY}}(x),
$$

and

$$
\beta W(x) \sim-A_{l l} \cos \left(\omega x+\varphi_{l l}\right) \exp (-\kappa x) .
$$

The last result has been obtained by Roth et al. [1] as well, through the linearization of equation (2.4) aiming to get the asymptotic behaviour. However, they have commented upon the fact that the asymptotic results (2.7) are in good agreement with density functional theory "at very large separations and, strikingly, at intermediate separations". Assuming the validity of the Derjaguin approximation, this is similar to our own observations $[2,11]$ regarding approximation (2.6), density functional theory, as well as some other theories. To comment on this, we wish to stress that the use of approximation (2.6) and expression (2.7) is not merely the result of a linearization that is reasonable at large separations. Its meaning has much deeper significance. The fact that expressions (2.5)-(2.7) are accurate, being constructed from the Percus-Yevick result $h_{l l}^{\mathrm{PY}}$, can be seen from their consistency with the experimental observation [10] that $W \sim R$ for very large spheres in low concentrations.

The expressions (2.6) and (2.7) for the potential of mean force can be thought as resulting from the exact relation (2.4) if the considered system will be described by the set of Ornstein-Zernike equations with the Percus-Yevick approximation for the small-small and large-small Ornstein-Zernike counterparts together with the use of the hypernetted chain (HNC) approximation for the large-large Ornstein-Zernike equation. This may be designated the $\mathrm{PY} / \mathrm{PY} / \mathrm{HNC}$ approximation and is not to be confused with a full implementation of the HNC approximation ( $\mathrm{HNC} / \mathrm{HNC} / \mathrm{HNC}$ ) used by Attard and Patey [12], for example. The latter would not result in analytical expressions but would satisfy the observation: $W \sim R$. In contrast, a PY/PY/PY procedure together with exact relation (2.4), would yield $W \sim \ln R$, a result with an incorrect quantitative form and seriously in error. It is only the combination $\mathrm{PY} / \mathrm{PY} / \mathrm{HNC}$ with equation (2.4), or equivalently PY/PY/PY with equation (2.5), that is sensible. 


\section{Conclusions}

The force between two large spheres can be obtained by differentiation of the interaction potential with respect to separation. However, since differentiation in direct (real) space is related to multiplication by $s$ in Laplace space, a more compact expression and further generalizations can be easier obtained by using the Laplace transform formalism. In particular, the asymptotic behaviour of the force and, through the Derjaguin approximation, the decay of the interaction energy per unit area between two planar surfaces are again determined by the same zero of $P(s)$ and both have the form of equation (2.7) but with other amplitude and phase coefficients $[3,4]$. In the case of two planar walls (slit-like film), the important property is the disjoining pressure which can be obtained by differentiation of the energy per unit area with respect to separation. From multiplication of the Laplace transform of the pair correlation function between two large spheres by $s^{2}$, we again have the result of the form of equation (2.7) for disjoining pressure only with other amplitude and phase parameters. In as much as the Fourier transform is related to the Laplace transform with a complex argument, equivalent results can be obtained from the procedure of Roth et al.

\section{Acknowledgements}

DH and AT were supported in part by the NSF (Grant No CHE98-13729). DTW was supported in part by a DOE grant.

\section{References}

1. Roth R., Evans R., Dietrich S. // Phys. Rev. E, 2000, vol. 62, p. 5360.

2. Trokhymchuk A., Henderson D., Wasan D.T. // J. Colloid Interface Sci., 1999, vol. 210 , p. 320.

3. Wasan D.T., Nikolov A.D., Trokhymchuk A., Henderson D. - In: Encyclopedia of Surface Science. Edited by A. Hubbard. San Diego, Academic Press (in press).

4. Trokhymchuk A., Henderson D, Nikolov A., Wasan D.T. // Langmuir, 2001, vol. 17, No. 16 , p. 4940-4947.

5. Henderson D. // J. Colloid Interface Sci., 1988, vol. 121, p. 486.

6. Evans R., Leote de Carvalho R.J.F., Henderson J.R., Hoyle D.C. // J. Chem. Phys., 1994, vol. 100, p. 591.

7. Wertheim M.S. // Phys. Rev. Lett., 1963, vol. 10, p. 321.

8. Perry P., Throop G. J. // J. Chem. Phys., 1972, vol. 57, p. 1827.

9. Experimental measurement [10] for the force $F$ indicates that $F(x)=\partial W(x) / \partial x \sim$ $R$. Since exact relation (2.4), we obtain the conclusion that in the colloidal limit $\ln \left[1+h_{l l}(x)\right] \sim R$. In particular, at the contact $x=0$, the PY result is $1+h_{l l}^{\mathrm{PY}}(0)=$ $(R / \sigma) \frac{3}{2} \eta /(1-\eta)^{2}$ that would predict $W \sim \ln R$.

10. Israelachvili J. Intermolecular and Surface Forces. New York, Academic Press, 1992.

11. Henderson D., Sokolowski S., Wasan D.T. // J. Stat. Phys., 1997, vol. 89, p. 243; J. Phys. Chem., 1998, vol. B102, p. 3009. 
12. Attard P., Patey G.N. // J. Chem. Phys., 1990, vol. 92, p. 4970.

\title{
До взаємодії між великими сферами у флюїді малих твердих сфер
}

\author{
Д.Гендерсон ${ }^{1}$, Д.Т.Васан ${ }^{2}$, А.Трохимчук ${ }^{1,3}$
}

1 Факультет хімії і біохімії, Університет Брайхем Янг, Прово, UT 84602, США

2 Факультет хімічної інженерії та охорони навколишнього середовища, Іллінойський технологічний інститут, Чикаго, IL 60616, США

3 Інститут фізики конденсованих систем НАН України, 79011 Львів, вул. Свєнціцького, 1

Отримано 25 вересня 2001 р.

Вказано і прокоментовано еквівалентність асимптотичного виразу для енергії взаємодії між парою великих твердих сфер у флюїді малих твердих сфер, який був отриманий Росом та ін. [Phys. Rev. E, 2000, том 62, с. 5360], використовуючи Фур'є перетворення і виразу, який ми отримали [J. Colloid Int. Sci., 1999, том 210, с. 320], використовуючи перетворення Лапласа.

Ключові слова: колоїдні суспензії, взаємодія

PACS: $82.70 . D d, 61.20 . G y$ 
